\title{
SCIENTIFIC REP

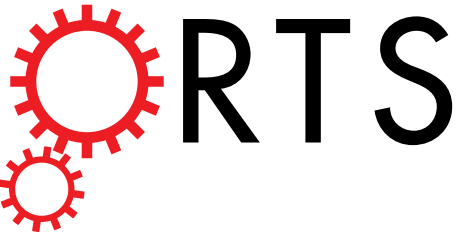 OPEN Fractionation for further conversion: from raw corn stover to lactic acid
}

Received: 25 August 2016

Accepted: 11 November 2016

Published: 05 December 2016

\author{
Ting He, Zhicheng Jiang, Ping Wu, Jian Yi, Jianmei Li \& Changwei Hu
}

Fractionation is considered to be one promising strategy to utilize raw biomass to its fullest and produce chemicals with high selectivity. Herein, ethanol/ $\mathrm{H}_{2} \mathrm{O}(1 / 1, \mathrm{v} / \mathrm{v})$ co-solvent with $0.050 \mathrm{M}$ oxalic acid is used to simultaneously fractionate $88.0 \mathrm{wt} \%$ of hemicellulose and $89.2 \mathrm{wt} \%$ of lignin in corn stover, while cellulose is not obviously degraded. $\mathrm{H}_{2} \mathrm{O}$ dissolves hemicellulose, $\mathrm{G}$ unit and those with $\beta-0-4$ linkage of lignin; whereas ethanol extracts $G$ and $S$ units as well as the skeleton with $\beta-5$ and $\beta-\beta$ linkages of lignin. Oxalic acid effectively catalyzes the hydrolysis of hemicellulose and breaks the intermolecular linkages between hemicellulose and lignin, therefore further promotes the release of lignin. The dissolved hemicelluloses derivatives are reprocessed to produce lactic acid obtaining a high yield of $79.6 \mathrm{wt} \%$ with $90 \%$ selectivity by the catalysis of $\mathrm{MgO}$. The remained cellulose and recovered lignin can be used further as feedstock to produce chemicals.

Recently, increasing interest has been focusing on the production of chemicals and fuels from renewable lignocellulosic biomass, due to the depletion of fossil resource and accompanying environmental pollution ${ }^{1-3}$. Hemicellulose, lignin and cellulose are the three main components of lignocelluloses. Utilization of the three main components without discard is of significant importance to fully exploit the potential of lignocellulose. Since these components exhibit distinct structure, numerous kinds of chemicals can be obtained from the simultaneous conversion of the three components in lignocelluloses ${ }^{4,5}$, leading to difficulties in their utilization, separation and further refinery. That is to say, the selectivity to each target product is quite low.

Fractionation provides ways to produce chemicals with high selectivity and to use the raw lignocellulose to its fullest ${ }^{6-10}$. Many fractionation methods have been developed for biorefinery concept, in most cases, aiming at providing carbohydrate fractions (cellulose and hemicellulose) for the following fermentation processes. In fact, lignin and hemicellulose are usually simultaneously extracted, leaving cellulose component apart, because the solvolysis of highly structured cellulose usually needs harsher conditions $s^{4,11,12}$. The lignocellulosic biomass can be then fractionated into its three components, which generate three feedstock streams for futher conversion. For the possibility to avoid harsh conditions (e.g. strong acids or alkaline) and complex processes, organic acid-catalyzed organosolv methods are promising to fractionate the three components in a more economical and environmentally friendly way ${ }^{13,14}$. Typically, a so-called "organocat process" was reported by Leitner et al., in which oxalic acid selectively catalyzed the depolymerization of hemicellulose and the dissolution of lignin in a 2 -methyltetrahydrofuran-water biphasic system ${ }^{15}$. This process released $>80 \%$ of hemicellulose as sugars from beech wood with $0.1 \mathrm{M}$ oxalic acid at $140^{\circ} \mathrm{C}$. The amount of recovered lignin reached up to $\sim 60-70 \%$ of the theoretical value from the average beech wood composition, whereas most of the cellulose was remained in solid pulp. However, the performance of both solvent and catalyst on the dissolution of the components is still unclear, which needs to be further investigated. Moreover, unlike cellulose and lignin, the fractionated hemicellulose derivatives by acid-catalyzed solovlysis is usually difficult to be separated to obtain solid intermediates, due to its further degradation into small molecular products. Thus, it is relatively hard to store and transport for further use as feedstock. Therefore, the prompt conversion of dissolved hemicellulose derivatives to chemicals with high selectivity after fractionation can be a way to avoid the problem.

Lactic acid, regarded as one of the top biomass derived platform chemicals by the US Department of Energy (DOE), has been widely used in food, pharmaceutical and chemical industries, especially in biodegradable polymer polylactic acid (PLA) production ${ }^{16-18}$. More than $90 \%$ of lactic acid are produced via fermentation of

Key Laboratory of Green Chemistry and Technology, Ministry of Education, College of Chemistry, Sichuan university, Chengdu, Sichuan 610064, China. Correspondence and requests for materials should be addressed to J.L. (email: lijianmei@scu.edu.cn)or C.H. (email: changweihu@scu.edu.cn) 


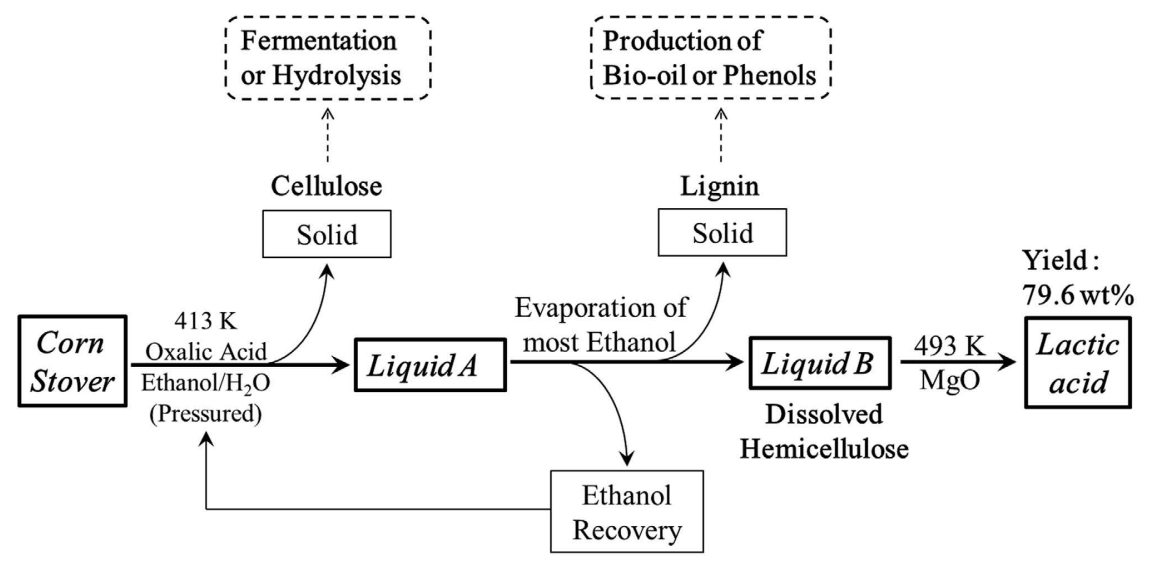

Figure 1. The schematic process for the fractionation of corn stover and the production of lactic acid.

carbohydrates, however, the biotechnological routes are high costly with low productivity ${ }^{17,19,20}$. In addition, the bioconversion process cannot be directly applied to the more available actual biomass without pretreatment, because of the inhibition of lignin ${ }^{21,22}$. At present, many efforts have been transferring to explore new chemo-catalytic methods in the interest of establishing a less expensive route for producing lactic acid ${ }^{23-25}$. Fangming Jin and coworkers obtained lactic acid ( $<42 \%$ carbon yield) from cellulose in the presence of alkaline ${ }^{26,27}$. Yanliang Wang and coworkers obtained a yield of lactic acid $(68 \mathrm{wt} \%)$ from cellulose in the presence of $7 \mathrm{mM} \mathrm{Pb}(\mathrm{II})^{28}$. $\mathrm{ErCl}_{3}$ showed an excellent catalytic activity to produce lactic acid from pure cellulose with a high yield of $91.9 \%$ (carbon yield) ${ }^{29}$. Although satisfied yield of lactic acid is obtained, these homogeneous catalysts somehow encounter the environment pollution and recycle problems, which hinders their application in industry. Heterogeneous catalyst therefore becomes an appropriate alternative. Glucose and cellulose (with $\mathrm{C}_{6}$ sugars units) are commonly used as the resources to produce lactic acid. To date, the hemicelluloses (mainly with $\mathrm{C}_{5}$ sugar units), especially those from actual lignocellulose, are seldom employed directly to produce lactic acid. Hongfei Lin et al. Reported that xylose and xylan could be used as resources to produce lactic acid by the catalysis of $\mathrm{ZrO}_{2}$. However, the highest yield of lactic acid was only $42 \mathrm{~mol} \%$ from xylose ${ }^{30}$. Actually, the hemicellulose via pretreatment process is a potential material to produce chemicals ${ }^{31-35}$. In this work, we therefore developed an integrated route to efficiently separate the three components of corn stover under mild conditions and prepare lactic acid from the derived-hemicellulose with high yield and selectivity.

\section{Results and Discussion}

A novel integrated route was developed to successfully fractionate corn stover into its three main components, and to convert the hemicellulose derivatives selectively to lactic acid (as shown in Fig. 1). This approach firstly disentangled both hemicellulose and lignin from corn stover in ethanol/ $\mathrm{H}_{2} \mathrm{O}$ co-solvent with oxalic acid as catalyst. After cellulose pulp and lignin precipitate were orderly separated, the dissolved hemicellulose derivatives in aqueous solution was selectively converted to lactic acid by the catalysis of $\mathrm{MgO}$.

Simultaneous dissolution of hemicellulose and lignin. Our initial experiments aimed to explore the effect of solvent (ethanol and $\mathrm{H}_{2} \mathrm{O}$ ) on the conversion of corn stover in the absence of catalyst (Entry 1-5 in Table 1). We observed that the conversion of hemicellulose in hot pressurized water (initial pressure of $\mathrm{N}_{2}$ was $2 \mathrm{MPa}$ before heating) reached up to $44.5 \mathrm{wt} \%$ at $140^{\circ} \mathrm{C}$ (Entry 1 in Table 1). Hot pressurized water could selectively extract hemicellulose (autohydrolysis process), not only playing an acid catalytic role to cut the links between hemicellulose and lignin as well as between carbohydrates, but also dissolving the hemicellulose fragments. A little amount of xylose and acetic acid were detected by HPLC. It indicated that water also partly broke the intramolecular linkages in hemicellulose fragments, resulting in the generation of small molecular compounds. Less water in ethanol/ $\mathrm{H}_{2} \mathrm{O}$ co-solvent led to lower conversion of hemicellulose. In agreement with our previous report ${ }^{36}$, the presence of ethanol in the system was beneficial to lignin dissolution. When the concentration of ethanol $\left(\mathrm{CE}\right.$, volume concentration of ethanol in $\left.\mathrm{H}_{2} \mathrm{O}\right)$ increased from 0 to 0.5 , the conversion of lignin increased from 27.5 to $38.6 \mathrm{wt} \%$. However, the decrease of lignin release was observed when the CE was more than 0.5 . Only $16.9 \mathrm{wt} \%$ of lignin was dissolved in ethanol, which was obviously lower than that obtained in water and ethanol $/ \mathrm{H}_{2} \mathrm{O}(1 / 1, \mathrm{v} / \mathrm{v})$ co-solvent systems. It indicated that when a part of hemicellulose (15.8 44.5 wt\%) depolymerized with $\mathrm{CE}<0.7$, the linkages between hemicellulose and lignin were broken, thereby lignin was easily released from biomass matrix.

Without catalyst, the small molecular products derived from hemicellulose and lignin were hardly observed after treatments, suggesting that the released hemicellulose and lignin were almost oligomers. Figure 2 shows the 2D HSQC of liquid fractions obtained from different solvent systems with varied CE in the absence of catalyst (reactions of Entry 1-5 in Table 1). Supplementary Table S1 lists the attribution of the main cross-signals according to literature ${ }^{36,37}$, and the hypothetical structure of lignin-derived species is displayed in Fig. S1. The signals of lignin oligomers and derivatives from hemicellulose were observed. $\mathrm{In}_{2} \mathrm{O}(\mathrm{CE}=0)$, the signals of xylan were observed obviously, and only $\mathrm{G}$ unit (guaiacyl unit) with A linkage ( $\beta-\mathrm{O}-4$ linkage) of lignin could be easily dissolved. This further proved that $\mathrm{H}_{2} \mathrm{O}$ was favorable for hemicellulose dissolution. In the presence of ethanol, the 


\begin{tabular}{|l|c|c|c|c|c|}
\hline \multirow{2}{*}{ Entry } & \multicolumn{2}{|c|}{ Conditions } & \multicolumn{3}{c|}{ Conversion of corn stover ${ }^{[\mathbf{b}]}$} \\
\cline { 2 - 6 } & $\mathbf{C E}^{[\mathbf{c}]}$ & $\mathbf{C O A}^{[\mathbf{c}]}$ & Cellulose & Hemicellulose & Lignin \\
\hline 1 & 0.0 & 0.000 & 0.0 & 44.5 & 27.5 \\
\hline 2 & 0.3 & 0.000 & 2.9 & 23.4 & 28.5 \\
\hline 3 & 0.5 & 0.000 & 0.4 & 15.8 & 38.6 \\
\hline 4 & 0.7 & 0.000 & 0.0 & 7.1 & 29.5 \\
\hline 5 & 1.0 & 0.000 & 0.0 & 0.0 & 16.9 \\
\hline 6 & 1.0 & 0.050 & 0.0 & 2.3 & 15.2 \\
\hline 7 & 0.0 & 0.050 & 12.0 & 98.1 & 47.0 \\
\hline 8 & 0.5 & 0.005 & 3.0 & 65.5 & 63.3 \\
\hline 9 & 0.5 & 0.010 & 5.1 & 83.2 & 77.6 \\
\hline 10 & 0.5 & 0.017 & 6.0 & 84.0 & 83.0 \\
\hline 11 & 0.5 & 0.033 & 6.7 & 86.7 & 87.1 \\
\hline 12 & 0.5 & 0.050 & 7.8 & 88.0 & 89.2 \\
\hline 13 & 0.5 & 0.067 & 14.1 & 91.5 & 89.4 \\
\hline
\end{tabular}

Table 1. Effect of different reaction conditions on the conversion of corn stover ${ }^{[\mathrm{a}]}{ }^{[\mathrm{a}]}$ The corn stover contains $45 \mathrm{wt} \%$ of cellulose, $21 \mathrm{wt} \%$ of hemicellulose, $17 \mathrm{wt} \%$ of lignin, $9 \mathrm{wt} \%$ of extractives, $3 \mathrm{wt} \%$ of ash, and $5 \mathrm{wt} \%$ of unknown composition. Reaction conditions: $3.0 \mathrm{~g}$ corn stover, $100 \mathrm{~mL}$ solvent, $140^{\circ} \mathrm{C}, 1 \mathrm{~h}$, initial pressure $2 \mathrm{MPa} \mathrm{N}_{2} \cdot{ }^{[\mathrm{b}]}$ Conversion percentage of the component based on its mass content in raw corn stover (wt\%). ${ }^{[\mathrm{c}]}$ Abbreviations used: $\mathrm{CE}=$ the concentration of ethanol in water $(\mathrm{v} / \mathrm{v}) ; \mathrm{COA}=$ the concentration of oxalic acid in $100 \mathrm{~mL}$ solvent $(\mathrm{M})$.

signals of S (syringyl unit) and $\mathrm{E}$ (cinnamate structure) were found. Both $\mathrm{G}$ and $\mathrm{S}$ units increased with increasing $\mathrm{CE}$. Moreover, in the aliphatic $\mathrm{C}-\mathrm{H}$ correlation region, the signals of A linkage gradually increased; and the signals of $C(\beta-5$ linkage) and $B(\beta-\beta$ linkage) appeared and gradually strengthened, indicating that the units with $\beta-5$ and $\beta$ - $\beta$ linkages were dissolved with the addition of ethanol. Therefore, we considered that the improved dissolution of lignin could be partly ascribed to the better dissolubility of lignin $\mathrm{G}$ and $\mathrm{S}$ units as well as those with $\mathrm{A}$, $\mathrm{B}$ and $\mathrm{C}$ linkages in ethanol/ $\mathrm{H}_{2} \mathrm{O}$ co-solvent than in $\mathrm{H}_{2} \mathrm{O}$. However, the signal of p-hydroxyphenyl unit $(\mathrm{H})$ was not observed, implying that it was hard to dissolve $\mathrm{H}$ unit in the absence of catalyst. In addition, according to the FTIR spectra of solid samples ( $\boldsymbol{c}$ and $\boldsymbol{e}$ in Fig. 3B), we found that the intensity of peaks at 1605 and $1515 \mathrm{~cm}^{-1}$ decreased, which indicated that ethanol in the system greatly affected the dissolution of lignin skeletal. If only water was used as solvent, it was hard to realize the collapse of lignin skeletal even in the presence of oxalic acid ( $\boldsymbol{b}$ and $\boldsymbol{d}$ in Fig. 3B). So, it seems that the skeletal is one of the stubborn and difficultly dissolved parts in lignin, and ethanol provides good ability for the extraction of lignin skeletal chunks. It explained why the available dissolution of lignin successfully proceeded only in the presence of ethanol. In general, water in the extraction medium firstly broke the links among the three components, then dissolved hemicellulose derivatives and G-type lignin unit, and guaranteed the transport of ethanol into the lignocellulosic matrix, thereby enabling lignin extraction. Ethanol effectively extracted the lignin (mainly focused on lignin skeleton) and was helpful for dissolving lignin-derivatives.

Oxalic acid, as a dicarboxylic acid, has attracted increasing interests because of its good catalytic ability for polysaccharide depolymerization ${ }^{15,38,39}$. With the addition of $0.017 \mathrm{M}$ oxalic acid, the conversion of hemicellulose and lignin greatly increased from 15.8 to $84.0 \mathrm{wt} \%$ and 38.6 to $83.0 \mathrm{wt} \%$, respectively. In order to investigate the performance of oxalic acid on the hemicellulose and lignin conversion more detailedly, lower concentrations of oxalic acid $(0.005$ and $0.010 \mathrm{M})$ were also tested. We found that when it was less than $0.017 \mathrm{M}$, the concentration of oxalic acid had an obvious effect on the conversion of both hemicellulose and lignin. With further increase of oxalic amount, the conversion of both hemicellulose and lignin only slightly improved. Therefore, the addition of oxalic acid greatly improved the extraction efficiency of both hemicellulose and lignin in corn stover, especially in ethanol $/ \mathrm{H}_{2} \mathrm{O}$ co-solvent. However, the cross-signals of $\mathrm{H}$ unit (in lignin) appared only when the concentration of oxalic acid was more than $0.033 \mathrm{M}$, and increased with the concentration of oxalic acid (Supplementary Fig. S2). Due to its poor dissolubility, $\mathrm{H}$ unit seems to be dissolved only in the presence of relatively a large amount of oxalic acid. Although the extraction efficiency of both hemicellulose and lignin was satisfied, higher concenteation $(>0.033 \mathrm{M})$ of oxalic acid was tested, for the purpose of releasing the $\mathrm{H}$ unit. Approximately $90 \mathrm{wt} \%$ of both hemicellulose and lignin could be finally extracted in ethanol/ $\mathrm{H}_{2} \mathrm{O}(1 / 1, \mathrm{v} / \mathrm{v})$ co-solvent with $0.050 \mathrm{M}$ oxalic acid at $140^{\circ} \mathrm{C}$ for $1 \mathrm{~h}$ (Entry $11-13$ in Table 1$)$, while no significant degradation of cellulose $(<8 \mathrm{wt} \%)$ was attained. To simultaneously convert hemicellulose and lignin while keep cellulose as much as possible, the reaction temperature and reaction time were optimized (Supplementary Fig. S3). It was obvious that the conversion of the three components increased with rising temperature. When the temperature was raised from 100 to $140^{\circ} \mathrm{C}$, the conversion of both hemicellulose and lignin increased rapidly from approximately 40 to $90 \mathrm{wt} \%$, while the conversion of cellulose slightly increased from 5 to $8 \mathrm{wt} \%$. Higher temperature than $140^{\circ} \mathrm{C}$ obviously boosted the conversion of cellulose from $8 \mathrm{wt} \%$ at $140^{\circ} \mathrm{C}$ to $15 \mathrm{wt} \%$ at $180^{\circ} \mathrm{C}$ (as shown in Supplementary Fig. S3A). The bands at 1427,1376 and $897 \mathrm{~cm}^{-1}$ in FTIR spectra of solid residue after treatment at $160^{\circ} \mathrm{C}$, corresponding to the crystalline structure of cellulose, obviously decreased, implying the depolymerization of cellulose in crystalline regions (Supplementary Fig. S4) ${ }^{40}$. These results agreed well with the literature reporting that dicarboxylic acids could selectively remove amorphous hemicellulose without extensive cellulose degradation when the pretreated 
।
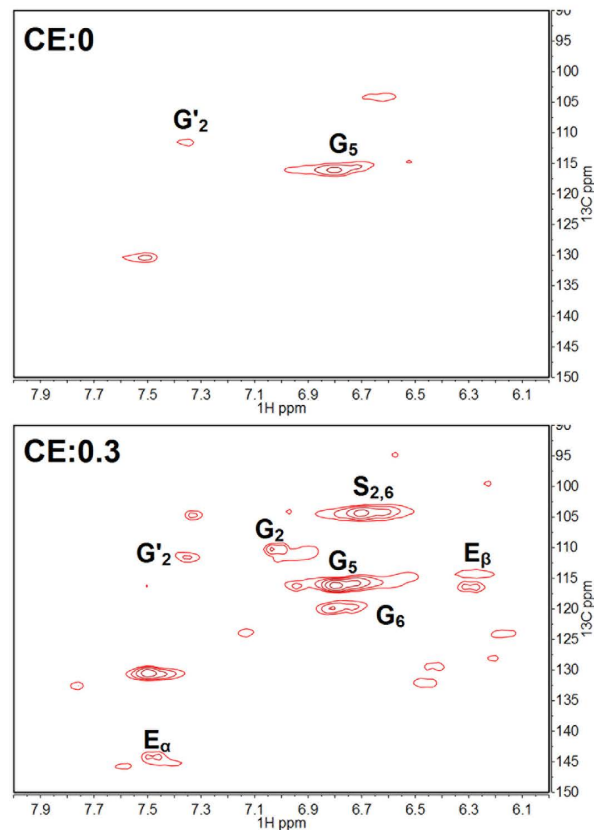

CE: 0.5
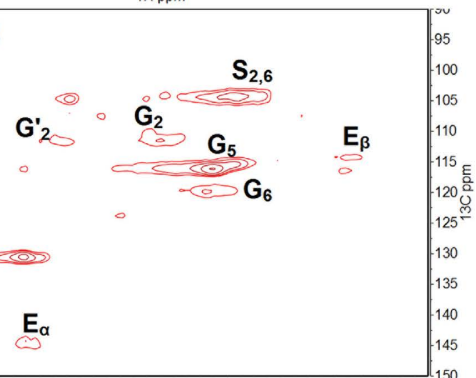

\begin{tabular}{lllllllllllll}
\hline 7.9 & 7.7 & 7.5 & 7.3 & 7.1 & 6.9 & 6.7 & 6.5 & 6.3 & 6.1 & 150 \\
\hline
\end{tabular}
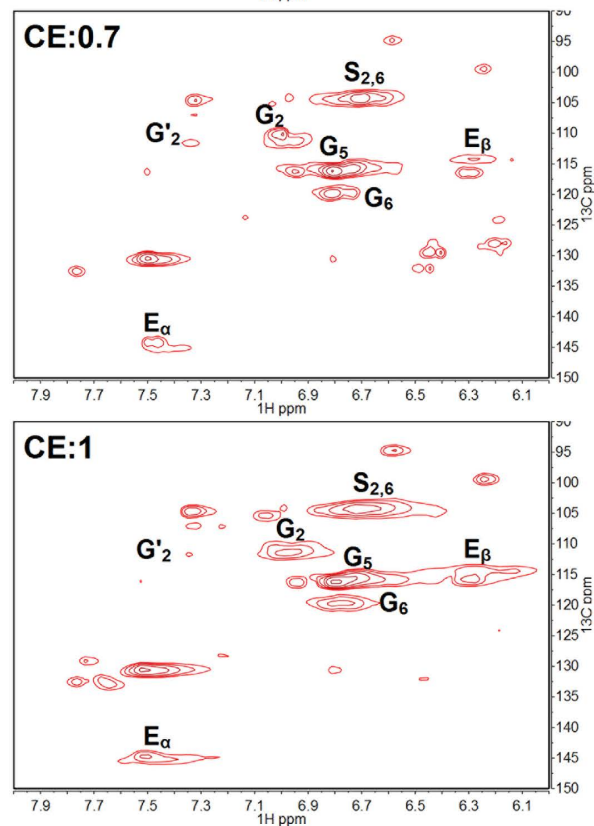

II
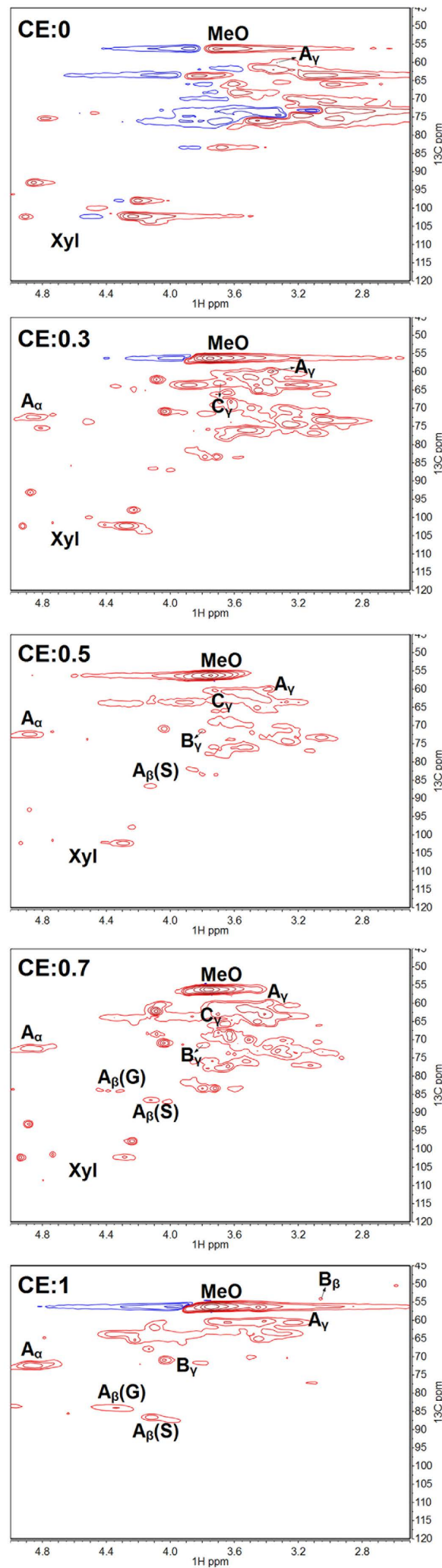

Figure 2. The 2D HSQC NMR analysis of liquid fraction obtained from different reaction system with various concentration of ethanol in $\mathrm{H}_{2} \mathrm{O}$. (I) Aromatic $\mathrm{C}-\mathrm{H}$ correlation region; (II) Aliphatic C-H correlation region. $\mathrm{CE}=$ the concentration of ethanol in ethanol $/ \mathrm{H}_{2} \mathrm{O}$ solvent; $\mathrm{A}: \beta-\mathrm{O}-4$ linkage; $\mathrm{B}$ : $\beta-\beta$ linkage; $\mathrm{C}: \beta-5$ linkage; E: cinnamate structure; G: guaiacyl units; S: syringly units; MeO: methoyls; Xyl: xylan derived from hemicellulose. 

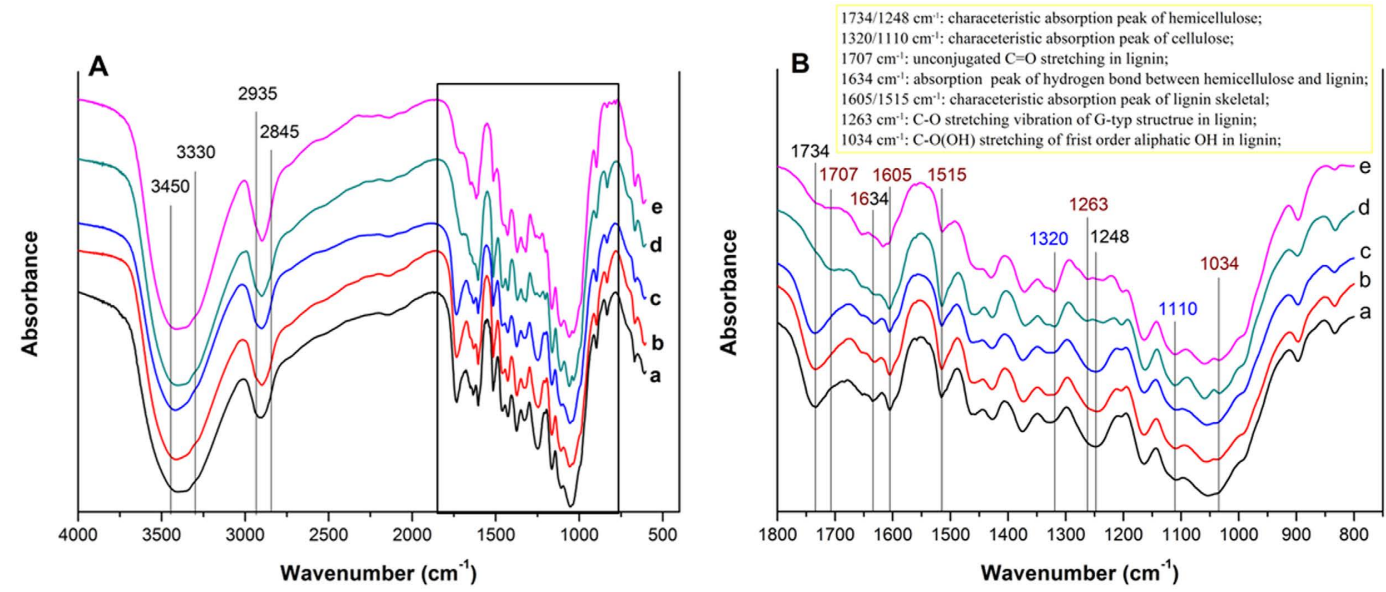

Figure 3. FTIR spectra of solid samples. (A) Full spectrum from $4000-600 \mathrm{~cm}^{-1}$; (B) Partial spectrum from $1800-800 \mathrm{~cm}^{-1}$. (a) Corn stover; (b) $\mathrm{H}_{2} \mathrm{O}, 0 \mathrm{M}$ oxalic acid; (c) ethanol/ $\mathrm{H}_{2} \mathrm{O}=1 / 1,0 \mathrm{M}$ oxalic acid; (d) $\mathrm{H}_{2} \mathrm{O}$, $0.050 \mathrm{M}$ oxalic acid; (e) ethanol $/ \mathrm{H}_{2} \mathrm{O}=1 / 1,0.050 \mathrm{M}$ oxalic acid.

temperature ranged in $80-140^{\circ} \mathrm{C}$, while the cellulose in crystalline regions would be depolymerized at temperature higher than $160^{\circ} \mathrm{C}^{15,38,41}$. Similarly, longer reaction time obviously promoted the conversion of cellulose (as shown in Supplementary Fig. S3B). Therefore, we chose $0.050 \mathrm{M}$ oxalic acid, $140^{\circ} \mathrm{C}$ and $1 \mathrm{~h}$ as the optimal reaction conditions.

The raw material and solid residues were characterized by FTIR spectroscopy. The stretching vibrations of $\mathrm{OH}, \mathrm{CH}_{3}$ and $\mathrm{CH}_{2}$ groups in the three main components of corn stover occur at 3450-3300, 2935 and $2845 \mathrm{~cm}^{-1}$, respectively (Fig. 3A). The main difference of FTIR spectra was in the regions ranged from $1800-800 \mathrm{~cm}^{-1}$ (Fig. 3B). In the presence of oxalic acid ( $\boldsymbol{d}$ and $\boldsymbol{e}$ ), the peaks at 1320 and $1110 \mathrm{~cm}^{-1}$ (assigned to cellulose) increased, while the peaks at 1734 and $1248 \mathrm{~cm}^{-1}$ (assigned to hemicellulose ) and those at 1707 and $1263 \mathrm{~cm}^{-1}$ (corresponding to lignin ) obviously weakened. This showed that the significant improvement of cellulose content in residues as well as the dissolution of both hemicellulose and lignin from corn stover was attributed to the presence of oxalic acid. The weakened peaks at $1034 \mathrm{~cm}^{-1}$ (attributing to $\mathrm{C}-\mathrm{O}(\mathrm{H})$ stretching vibration of the first order aliphatic $\mathrm{OH}$ in lignin $)^{42}$ and $1634 \mathrm{~cm}^{-1}$ (attributing to a hydrogen bond between hemicellulose and lignin) ${ }^{43}$ suggested the damage of hydrogen bond between hemicellulose and lignin, resulting from the penetration of oxalic acid in the amorphous region of the biomass ${ }^{44}$. With $0.050 \mathrm{M}$ oxalic acid, $47.0 \mathrm{wt} \%$ of lignin was degraded in water (Entry 7 in Table 1). Most of the G-type lignin (at $1263 \mathrm{~cm}^{-1}$ ) was removed according to $d$ of Fig. 3B. In addition, we could find obvious signals of $G$ and $S$ units as well as those with A linkage from its 2D HSQC (Supplementary Fig. S5), indicating the release of G and S lignin with A linkage. It seemed that the G-type lignin was more easily released from biomass matrix, and S-type lignin tended to be released by the catalysis of oxalic acid in $\mathrm{H}_{2} \mathrm{O}$. We considered that the first order aliphatic $\mathrm{C}-\mathrm{OH}$ in lignin was intimately related to the formation of the hydrogen bond between hemicellulose and lignin. The oxalic acid led to the breakage of the hydrogen bond, effectually contributing to the dissolution of G lignin. Therefore, the facilitating of the conversion of both hemicellulose and lignin by oxalic acid could be ascribed to the breakage of interaction between them. Control experiments with different catalysts (Supplementary Fig. S6) showed that other acids, including inorganic acid (hydrochloric acid) and organic acids (butanedioic acid, formic acid, acetic acid and maleic acid), with approximately the same initial $\mathrm{pH}$ value as oxalic acid, resulted in close conversion of both hemicellulose and lignin. Potassium oxalate with the same concentration of oxalate exhibited poor catalytic activity for the conversion of both hemicellulose and lignin. Therefore, moderate acidity $(\mathrm{pH}=2)$ of oxalic acid in the system was the main factor that resulted in the simultaneous dissolution of the hemicellulose and lignin without significantly affecting the cellulose component.

Separation of the three components. Under the cooperative action of catalyst and solvent, the cellulose in corn stover lost the protecting shield of hemicellulose and lignin (Supplementary Fig. S7A). It would be more available for biodegradation by cellulolytic enzymes. The majority of cellulose was remained in residue with a purity of $\sim 70 \mathrm{wt} \%$. Because the removed hemicellulose and lignin were in amorphous region, the CI (crystalline) of resulted cellulose was increased from 79\% to $88 \%$ (Supplementary Fig. S7B). Nevertheless, the majority (80\%) of "others" in corn stover, including ash, extractives and unknown composition, was retained in residue with cellulose. After evaporation of ethanol from the liquid (Liquid A, referring to Fig. 1), a yield of $83 \mathrm{wt} \%$ (based on converted lignin) lignin (a rufous powder) could be recovered. Its 2D HSQC NMR spectra (Supplementary Fig. S8) showed that the main construction unit of lignin was remained. The separated lignin was dsisolved in THF and analyzed by GPC. Its $\mathrm{M}_{\mathrm{w}}\left(1480 \mathrm{~g} \mathrm{~mol}^{-1}\right)$ was much lower than that in natural raw material, resulting from the fractionation and dissolution treatments. However, the relevant small molecular products (e.g. monophenic compounds) derived from lignin was hardly detected. Thus, it indicated that the removed lignin was mainly degradated to oligomers ( $\sim 10$ benzene propane units) without any monomers. Many small molecular products were detected in hemicellulose fraction (Liquid B) (Fig. 4), including xylose, lactic acid, some $(<9 \mathrm{wt} \%)$ formic acid (FA) and acetic acid (AA), while furfural was rarely observed. The yields of lactic acid and xylose were 


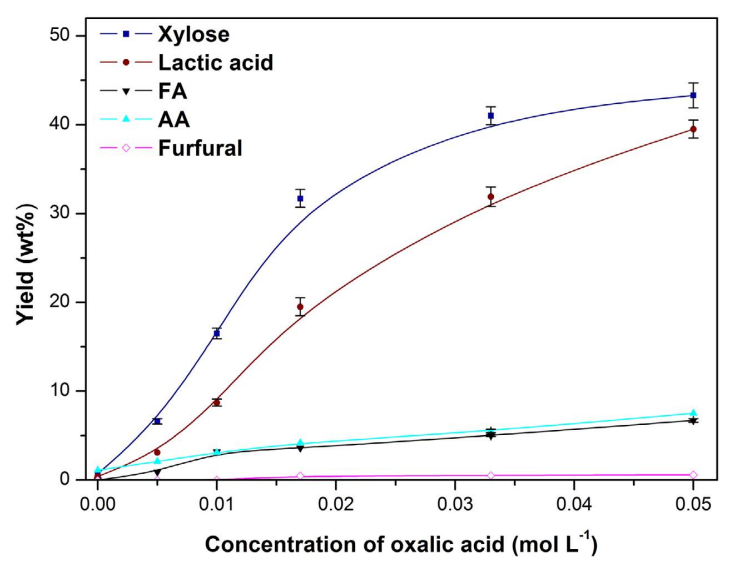

Figure 4. The yield of main monomers with different concentration of oxalic acid. Reaction conditions: $3.0 \mathrm{~g}$ corn stover, ethanol $/ \mathrm{H}_{2} \mathrm{O}(1 / 1, \mathrm{v} / \mathrm{v}) 100 \mathrm{~mL}, 140^{\circ} \mathrm{C}$. $1 \mathrm{~h}$, initial pressure $2 \mathrm{MPa} \mathrm{N}$.

\begin{tabular}{|l|c|c|c|c|c|c|}
\hline \multirow{2}{*}{$\begin{array}{l}\text { Temperature } \\
\left({ }^{\circ} \mathbf{C}\right)\end{array}$} & \multicolumn{5}{|c|}{ Yield wt\% } & \multirow{2}{*}{} \\
\cline { 2 - 6 } & Xylose & $\begin{array}{c}\text { Lactic } \\
\text { acid }\end{array}$ & FA & AA & Furfural & SL $^{[\mathrm{b}]}$ \\
\hline Liquid B & 43.3 & 39.5 & 6.7 & 7.5 & 0.6 & 45 \\
\hline $160^{[\mathrm{a}]}$ & 50.9 & 44.9 & 11.6 & 7.9 & 2.7 & 51 \\
\hline $180^{[\mathrm{a}]}$ & 34.9 & 39.0 & 12.6 & 9.5 & 8.4 & 44 \\
\hline $220^{[\mathrm{a}]}$ & 1.2 & 14.5 & 8.8 & 11.2 & 21.0 & 16 \\
\hline
\end{tabular}

Table 2. The yield of main liquid products and the selectivity to lactic acid in Liquid B and further reaction at different temperature. ${ }^{[a]}$ Reaction conditionns: $1 \mathrm{~h}$ without the addition of catalysts, initial pressure $2 \mathrm{MPa}$ $\mathrm{N}_{2} \cdot{ }^{[\mathrm{b}]} \mathrm{SL}=$ the selectivity to lactic acid (\%).

greatly improved with increasing concentration of oxalic acid, and $43.3 \mathrm{wt} \%$ of xylose and $39.5 \mathrm{wt} \%$ of lactic acid were obtained in the presence of $0.050 \mathrm{M}$ oxalic acid. It is commonly accepted that xylan chain is firstly decomposed to xylo-oligosaccharides, and then the decomposition proceeds to yield xylose monomer ${ }^{45}$. Increasing the concentration of oxalic acid promoted the further degradation of xylo-oligosaccharides, and the resulted xylose could be further converted to other smaller molecular products like lactic acid.

Enhancement of lactic acid production. Besides $39.5 \mathrm{wt} \%$ of lactic acid, $43.3 \mathrm{wt} \%$ of xylose was also contained in Liquid B (Table 2). We afterwards tried to further increase the yield of lactic acid through reprocessing the aqueous hemicellulose derivatives. Primarily, Liquid B was directly heated to higher temperature $(160,180$ and $220^{\circ} \mathrm{C}$ ), and the results were shown in Table 2 . At $160^{\circ} \mathrm{C}$, the yield of both xylose and lactic acid increased to $50.9 \mathrm{wt} \%$ and $44.9 \mathrm{wt} \%$, respectively. It implied that the appropriate increase in temperature was advantageous for the further hydrolysis of xylo-oligosaccharides, resulting in the increase of xylose yield. This further proved the presence of xylo-oligosaccharides from hemicellulose conversion. The main by-products, including FA, AA and furfural, were also slightly increased. However, with further raising reaction temperature to $220^{\circ} \mathrm{C}$, the yield of both xylose and lactic acid decreased to $1.2 \mathrm{wt} \%$ and $14.5 \mathrm{wt} \%$, respectively; while the yield of furfural significantly increased from 0.6 to $21.0 \mathrm{wt} \%$. This was attributed to the dehydration of xylose to furfural at high temperatures under BrØnsted acidic ambiance, and the further decomposition of lactic acid to smaller molecular acids $(\text { like FA and AA) })^{30}$.

Known that lactic acid was the main product from sugars by the catalysis of alkaline catalyst ${ }^{24}$, we next introduced commercially accessible $\mathrm{MgO}$ (with a surface area of $34 \mathrm{~m}^{2} \mathrm{~g}^{-1}$ ) to reprocess Liquid B. CO 2 TPD results showed that a large amount of weakly basic sites and some medium/strong basic sites existed on $\mathrm{MgO}$ (Supplementary Fig. S9). It is believed that the weakly basic sites are the catalytic active sites, and more weakly sites there are, higher catalytic activity the MgO has ${ }^{46}$. SEM and TEM were also employed to characterize the structure of MgO (Supplementary Figs S10 and S11). In general, nanosheets of the MgO gather together with some nanorods insertion. In the presence of $\mathrm{MgO}$, xylose was completely converted, and the yield and selectivity to lactic acid were significantly improved at $160^{\circ} \mathrm{C}$ (Fig. 5). The yield of lactic acid increased to $48.6 \mathrm{wt} \% \mathrm{with}$ $0.1 \mathrm{~g} \mathrm{MgO}$. Further increase of $\mathrm{MgO}$ amount led to obvious enhancement of lactic acid yield, which reached to $59.7 \mathrm{wt} \%$ when $0.2 \mathrm{~g} \mathrm{MgO}$ was used. A high yield (68.4 wt\%) and selectivity (78\%) to lactic acid were achieved with $0.5 \mathrm{~g} \mathrm{MgO}$. However, further increase of $\mathrm{MgO}$ amount did not have an obvious contribution to the improvement of lactic acid yield. Note worthily, furfural was hardly detected in the presence of $\mathrm{MgO}$. It was considered that $\mathrm{MgO}$ might change the acidic ambiance of the reaction system, so that the formation of furfural was inhibited, whereas the conversion of xylose to lactic acid was effectively facilitated.

As the reaction time extended, the yield of lactic acid initially increased but decreased afterwards at $160^{\circ} \mathrm{C}$ with $0.5 \mathrm{~g} \mathrm{MgO}$ (Fig. 6A). When $\mathrm{t}=0$ (stopping heating the reactor immediately as soon as it reached to the 


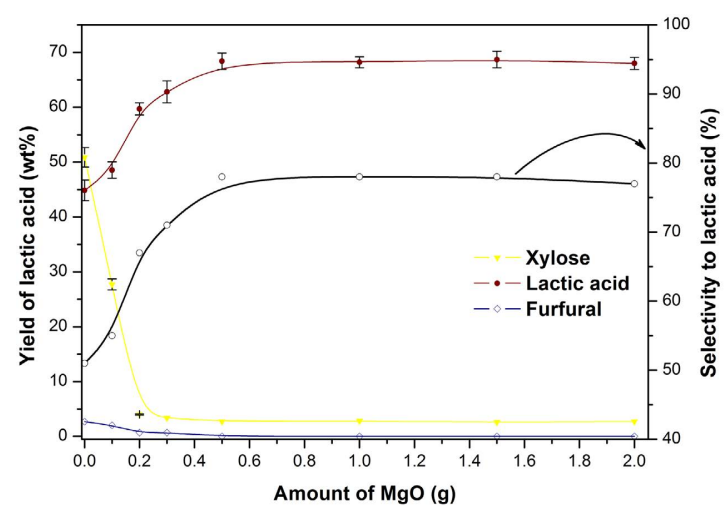

Figure 5. The influence of $\mathrm{MgO}$ amount on the yield of liquid products and selectivity to lactic acid. Reaction condition: $160^{\circ} \mathrm{C}, 1 \mathrm{~h}$, initial pressure $2 \mathrm{MPa} \mathrm{N}_{2}$.
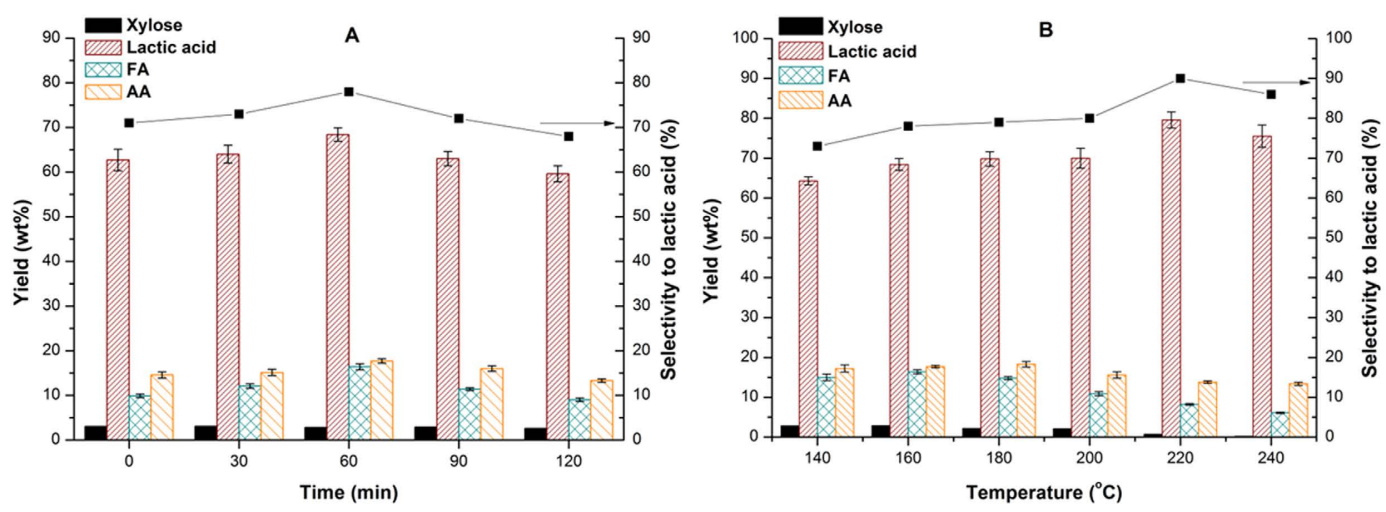

Figure 6. The effect of reaction time (A) and temperature (B) on the formation of lactic acid.

designed temperature), the yield of lactic acid was $62.7 \mathrm{wt} \%$, and the highest yield reached $68.4 \mathrm{wt} \%$ at $\mathrm{t}=1 \mathrm{~h}$. Futher increasing reaction time did not lead to further conversion of xylose with no obvious change in the yield of FA and AA. The production of lactic acid was further conducted at different reaction temperatures (Fig. 6B). When the reaction temperature was raised to $200^{\circ} \mathrm{C}$ from $140{ }^{\circ} \mathrm{C}$, the yield of lactic acid had a slight increase, from 64.3 to $69.8 \mathrm{wt} \%$. With further increase of temperature to $220^{\circ} \mathrm{C}$, nearly all the xylose was converted, and the yield and selectivity to lactic acid got the maximum, $79.6 \mathrm{wt} \%$ and $90 \%$, respectively. Thus, it was considered that $\mathrm{MgO}$ could inhibit the lactic acid from further conversion at higher temperature. However, a drop of lactic acid yield happened at $240^{\circ} \mathrm{C}$. Therefore, the reaction temperature should be maintained below $240^{\circ} \mathrm{C}$ to restrain further conversion of lactic acid.

We also studied the recyclability of MgO. As shown in Supplementary Fig. S12A, its catalytic activity for lactic acid production had no obvious loss after 5 runs. It indicated that commercial $\mathrm{MgO}$ indeed exhibited excellent recyclability. A little change of $\mathrm{MgO}$ was observed after reuse through XRD analysis (Supplementary Fig. S12B). Because of its advantages such as safety, low cost and easy preparation, $\mathrm{MgO}$ is a potential industrial catalyst for the production of lactic acid.

Discussion on the origin of lactic acid. In most cases, the conversion of hemicellulose or xylan to lactic acid firstly passes through the depolymerization of hemicellulose or xylan to xylose monomer in the presence of acid catalyst. Then, the xylose is further converted to glycolaldehyde and glyceraldehyde via a retro-aldol condensation reaction. Consequently, lactic acid is produced ${ }^{30,45,47}$. In this reaction pathway, the theoretical yield of lactic acid based on xylose can be calculated as $60 \mathrm{wt} \%$. In our work, the highest yield of lactic acid reached up to $79.6 \mathrm{wt} \%$ based on the mass of hemicellulose contained in corn stover, which was much higher than the theoretical yield. In order to investigate the origin of the high yield of lactic acid, Liquid B was analyzed by GPC. The results showed that there were still some oligomers $\left(\mathrm{M}_{\mathrm{w}}: 654 \mathrm{~g} \mathrm{~mol}^{-1}\right)$ in Liquid $\mathrm{B}$. Control experiments were therefore conducted using microcrystalline cellulose and cellobiose as the starting material, respectively. The yield of lactic acid derived from cellobiose was lower than $40 \mathrm{wt} \%$ (shown as Fig. 7), while lactic acid was hardly obtained from microcrystalline cellulose. Considering the fact that only $8 \mathrm{wt} \%$ of cellulose was converted, we believed that the majority of lactic acid was derived from hemicellulose. In addition, when other sugars contained in corn stover, including xylose, arabinose, glucose, glacatose and mannose, were employed as substrates under the same conditions of the second step $\left(0.5 \mathrm{~g} \mathrm{MgO}, 220^{\circ} \mathrm{C}\right.$ and $\left.1 \mathrm{~h}\right)$, the yield of lactic acid was less than $45 \mathrm{wt} \%$ (Fig. 7). These results indicated that cellobiose and other sugars contained in corn stover were not mainly 


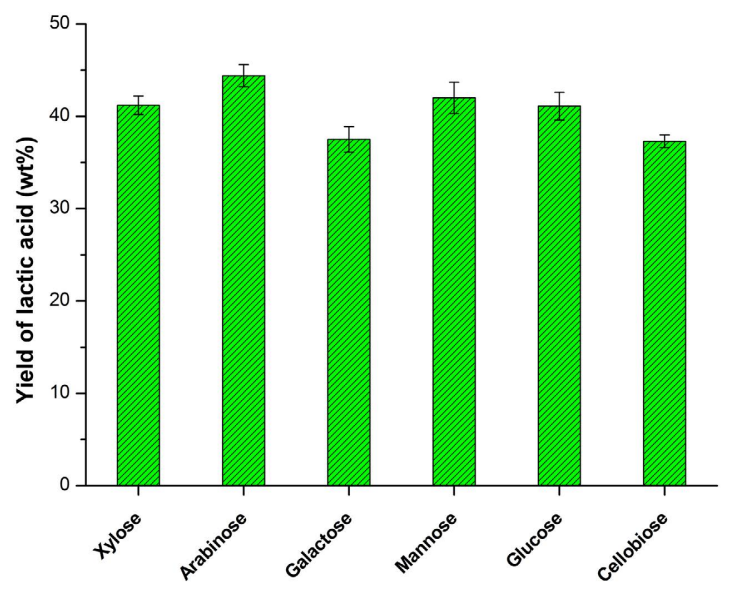

Figure 7. The yield of lactic acid derived from different sugars. Reaction condition: $0.63 \mathrm{~g}$ substrate, $0.5 \mathrm{~g}$ $\mathrm{MgO}, 220^{\circ} \mathrm{C}, 1 \mathrm{~h}$, initial pressure $2 \mathrm{MPa} \mathrm{N}_{2}$.

responsible for the high yield of lactic acid. Besides hemicellulosic/cellulosic oligomers, glucose and xylose, Liquid B also contained some lactic acid, FA and AA (Table 2) generated from the first step. It was therefore inferred that lactic acid, FA and AA might influence on the pathway of xylose conversion to lactic acid. Control experiments were also conducted, under the same conditions of the second step $\left(0.5 \mathrm{~g} \mathrm{MgO}, 220^{\circ} \mathrm{C}\right.$ and $\left.1 \mathrm{~h}\right)$, using xylose as substrate with the addition of equivalent quantity of FA, AA and lactic acid in reaction system, respectively. When the additive was FA or AA, it generated $<40 \mathrm{wt} \%$ of lactic acid. Unexpectedly, the addition of lactic acid significantly promoted the yield of lactic acid ( $>60 \mathrm{wt} \%)$, which was much higher than that without the addition of lactic acid (41 wt\%). However, in the absence of $\mathrm{MgO}$, the main product was $\sim 30 \mathrm{wt} \%$ of furfural using xylose as substrat with the addition of FA and AA, respectively; and when the additive was lactic acid, no newly generated lactic acid was obtained. It suggested that the added-lactic acid and $\mathrm{MgO}$ may exhibit synergistic effect on the unusually high yield of lactic acid. Of course, it is necessary to deeply investigate in future work. We therefore inferred that the formation of lactic acid in the second step might pass through some other pathways different from the typical reaction mechanism reported ${ }^{30}$, for example, the formation of lactic acid directly from oligomers (similar to the formation of glycol directly from cellulose oligomers reported by Tao Zhang et al..$^{48}$ ). In this case, the first step provided special feedstock for the second step to produce lactic acid with unusually high yield (79.6 wt $\%)$ catalyzed by $\mathrm{MgO}$.

\section{Conclusions}

We have achieved the fractionation of the three components in corn stover into aqueous hemicellulose derivatives, solid cellulose and solid lignin. The obtained hemicellulose derivatives, which was difficult to store and transport, was further converted to value-added chemical (lactic acid) with high yield (79.6 wt $\%$ ) and selectivity (90\%), through a well-designed chemo-catalytic biorefining process. Firstly, $\sim 90 \mathrm{wt} \%$ of the hemicellulose and lignin in corn stover were simultaneously dissolved in ethanol/ $\mathrm{H}_{2} \mathrm{O}(1 / 1, \mathrm{v} / \mathrm{v})$ co-solvent with $0.050 \mathrm{M}$ oxalic acid at $140{ }^{\circ} \mathrm{C}$ for $1 \mathrm{~h}$; while the cellulose was not obviously degraded and could be separated by simple filtration. Wherein, $\mathrm{H}_{2} \mathrm{O}$ dissolved hemicellulose, $\mathrm{G}$ unit and those with $\beta-\mathrm{O}-4$ linkage of lignin; while ethanol extracted $\mathrm{G}$ and $\mathrm{S}$ units as well as the skeleton with $\beta-5$ and $\beta-\beta$ linkages of lignin. Oxalic acid effectively catalyzed the hydrolysis of hemicellulose and broke the intermolecular linkages between hemicellulose and lignin, and therefore further promoted the release of lignin. Then, the released lignin was precipitated after removal of ethanol. The obtained solid cellulose and lignin may be suitable raw materials for bio-chemicals and bio-oil production. Finally, the dissolved hemicellulose derivatives, co-existed as a mixture including xylose, cellulosic/hemicellulosic oligomers and lactic acid, was used as feedstock to enhance the yield of lactic acid with $\mathrm{MgO}$ at $220^{\circ} \mathrm{C}$. The first step (fractionation) provided special feedstock for the second step to produce lactic acid with unusually high yield catalyzed by $\mathrm{MgO}$. MgO exhibited good recyclability. This comprehensive process may be very promising to effectively utilize the raw biomass to its fullest and produces target products with high selectivity.

\section{Method}

Materials. Corn stover was obtained from Fushun in Liaoning province of China. It was smashed by miniature plant sample mill $\left(1400 \mathrm{r} \mathrm{min}^{-1}, 180 \mathrm{~W}, 2 \mathrm{~g} \mathrm{~min}^{-1}\right)$. The size of corn stover powder ranged from $60-100$ mesh. It was dried in an oven at $100^{\circ} \mathrm{C}$ overnight before use. The chemicals used in this work were obtained from commercial sources and used without further purification except $\mathrm{MgO}$, which was calcined at $700^{\circ} \mathrm{C}$ for $4 \mathrm{~h}$ before use.

Simultaneous dissolution of hemicellulose and lignin in corn stover. The treatment of corn stover to simultaneously dissolve hemicellulose and lignin was performed in a $200 \mathrm{~mL}$ stainless steel autoclave equipped with a magnetic stirring device and a temperature controller. $3.0 \mathrm{~g}$ corn stover powder and designed amount of oxalic acid were added in the reactor with $100 \mathrm{~mL}$ ethanol/ $\mathrm{H}_{2} \mathrm{O}$ (typically $1 / 1, \mathrm{v} / \mathrm{v}$ ). Then nitrogen gas was bubbled into the autoclave for three minutes at a rate of $300-400 \mathrm{~mL} \mathrm{~min}^{-1}$, so that the interior air was completely replaced 
by $\mathrm{N}_{2}$. The initial pressure was kept at $2.0 \mathrm{MPa}$. The reactor was heated $\left(4^{\circ} \mathrm{C} \mathrm{min}{ }^{-1}\right)$ from room temperature to the target temperature and kept for designed reaction time (typically $1 \mathrm{~h}$ ). When the reaction was finished, the reactor was pulled out from the heating device and cooled down naturally to room temperature. After depressurizing the reactor, the mixture was fully poured out. The reactor was washed for three times using ethanol/ $\mathrm{H}_{2} \mathrm{O}$ $(1 / 1, v / v)$, and the solution was merged to the mixture. Then the solid residue was separated from liquid fraction by vacuum filtration, washed with ethanol $/ \mathrm{H}_{2} \mathrm{O}(1 / 1, \mathrm{v} / \mathrm{v})$ for three times and dried at $100^{\circ} \mathrm{C}$ overnight. The washing liquor and liquid fraction were mixed together (defined as Liquid A) and stored for subsequent processing.

Recovery of lignin. Rotary evaporator was used to evaporate ethanol from Liquid A. Distillation conditions: $34-37^{\circ} \mathrm{C}$, vacuum pressure $0.09 \mathrm{MPa}, 150 \mathrm{rpm}, 1 \mathrm{~h}$. Then the dissolved lignin oligomers were precipitated and filtered. After drying naturally, a reddish brown powder was obtained. The remained aqueous solution was defined as Liquid B.

Enhancement of lactic acid production. Liquid B was further subjected to enhance lactic acid production by the catalysis of $\mathrm{MgO}$. Designed amount of Liquid B and $\mathrm{MgO}$ were placed in a steel autoclave, which was then sealed and pressurized with $2.0 \mathrm{MPa} \mathrm{N}_{2}$. The reactor was heated $\left(4^{\circ} \mathrm{C} \mathrm{min}{ }^{-1}\right)$ from room temperature to the target temperature. After the reaction was finished, the reactor was pulled out from the heating device, and cooled down naturally to room temperature. The mixture in the reactor was poured out and filtered to recover the catalyst by vacuum filtration. The filter residue was washed with $15 \mathrm{~mL}$ water for 3 times. The filtrate and washing liquor were merged together and further characterized by HPLC. We performed the same experiment at least in triplicate for each run, and the standard error was $<5 \%$.

Characterization of the solid residue. Classical chemical titration methods were employed to analyze the three main components (cellulose, hemicellulose and lignin) in corn stover and in the reaction residue. The experimental details could be found in refs 36 and 49. The FTIR spectra of the solid samples were recorded on a Nicolet 6700 Fourier transform infrared spectrometer in the range of $4000-400 \mathrm{~cm}^{-1}$ with a resolution of $4 \mathrm{~cm}^{-1}$. $1 \mathrm{mg}$ dried samples were blended with $100 \mathrm{mg} \mathrm{KBr}$ and pressed into thin pellets before measurement.

Characterization of liquid products. Qualitative and quantitative analyses of the small molecular liquid products were performed on the Dionex Ultimate 3000 high performance liquid chromatograph (column HPX$87 \mathrm{H} 300 \times 7.8 \mathrm{~mm}$ ) with a RI detector. The mobile phase was $0.005 \mathrm{M}$ sulfuric acid aqueous solution with a flow rate of $0.6 \mathrm{~mL} \mathrm{~min}^{-1}$. The detection temperature of column and RI detector were $50^{\circ} \mathrm{C}$ and $35^{\circ} \mathrm{C}$, respectively. The total testing time was $55 \mathrm{~min}$. External standard method was employed to quantify the products, using calibration curves from authentic samples. The yield of liquid products and the selectivity to lactic acid reported in this work are defined as follow:

$$
\text { Yield }(\mathrm{wt} \%)=\frac{\text { liquid product amount }(\mathrm{g})}{\text { hemicellulose amount contained in corn stover }(\mathrm{g})} \times 100 \%
$$

$$
\text { Selectivity to lactic acid }(\%)=\frac{\text { lactic acid amount }(\mathrm{g})}{\text { converted hemicellulose amount in corn stover }(\mathrm{g})} \times 100 \%
$$

The weight-average molecular weights $\left(\mathrm{M}_{\mathrm{w}}\right)$ of liquid products and recovered lignin were analyzed using a gel permeation chromatograph (GPC, HLC-8320) with TSK gel Super HM-H $(6.0 \mathrm{~mm} \times 15 \mathrm{~cm} \times 2)$ columns and a RI detector. Both the columns and RI detector were maintained at $40^{\circ} \mathrm{C}$ during analysis. After removing solvents $\left(\mathrm{H}_{2} \mathrm{O}\right.$ and ethanol) from the reaction liquid fraction, a viscous liquid was obtained as the sample. THF was used to dissolve the sample (for both recovered lignin and viscous liquid) and also used as eluent with a flow rate of $0.6 \mathrm{~mL} \mathrm{~min}^{-1}$ for GPC analysis. An injection volume of $10 \mu \mathrm{L}$ was used. A calibration curve was obtained using monodisperse polystyrene standards. The 2D HSQC NMR spectra of liquid products from lignin were qualitatively determined on a Bruker Avance $400 \mathrm{MHz}$ spectrometer. About $50 \mathrm{mg}$ sample was fully dissolved in $0.5 \mathrm{~mL}$ deuterated dimethylsulfoxide (DMSO- $\left.\mathrm{d}^{6}\right)$. Heteronuclear single quantum coherence (HSQC) experiments were performed with 10 to $0 \mathrm{ppm}$ and 220 to $5 \mathrm{ppm}$ in ${ }^{1} \mathrm{H}$ - and ${ }^{13} \mathrm{C}$-dimensions, respectively. For the ${ }^{1} \mathrm{H}$-dimension, 1024 data points were used for an acquisition time of $64 \mathrm{~ms}$, while 256 data points were used for the ${ }^{13} \mathrm{C}$-dimension. The ${ }^{1} J_{\mathrm{C}-\mathrm{H}}$ used was $145 \mathrm{~Hz}$. Besides, $1.5 \mathrm{~s}$ pulse delay and 16 scans were also adopted. The DMSO solvent peak $\left(\delta_{\mathrm{C}} 39.5 \mathrm{ppm}\right.$ and $\left.\delta_{\mathrm{H}} 2.5 \mathrm{ppm}\right)$ was used for the chemical shift calibration.

\section{References}

1. Tuck, C. O., Pérez, E., Horváth, I. T., Sheldon, R. A. \& Poliakoff, M. Valorization of biomass: deriving more value from Waste. Science 337, 695-699 (2012).

2. Gallezot, P. Conversion of biomass to selected chemical products. Chem. Soc. Rev. 41, 1538-1558 (2012).

3. Somerville, C., Youngs, H., Taylor, C., Davis, S. C. \& Long, S. P. Feedstocks for lignocellulosic biofuels. Science 329, 790-792 (2010).

4. Corma, A., Iborra, S. \& Velty, A. Chemical routes for the transformation of biomass into chemicals. Chem. Rev. 107, 2411-2502 (2007).

5. Li, C., Zheng, M., Wang, A. \& Zhang, T. One-pot catalytic hydrocracking of raw woody biomass into chemicals over supported carbide catalysts: simultaneous conversion of cellulose, hemicellulose and lignin. Energy Environ. Sci. 5, 6383-6390 (2012).

6. Luterbacher, J. S. et al. Nonenzymatic sugar production from biomass using biomass-derived GVL. Science 343, 277-280 (2014).

7. Zhao, X., Cheng, K. \& Liu, D. Organosolv pretreatment of lignocellulosic biomass for enzymatic hydrolysis. Appl. Mmicrobiol. Biot. 82, 815-827 (2009).

8. Bosch, S. V. d. et al. Reductive lignocellulose fractionation into soluble lignin-derived phenolic monomers and dimers and processable carbohydrate pulps. Energy Environ. Sci. 8, 1748-1763 (2015).

9. Käldström, M., Meine, N., Farès, C., Rinaldi, R. \& Schüth, F. Fractionation of 'water-soluble lignocellulose' into $C_{5} / C_{6}$ sugars and sulfur-free lignins. Green Chem. 16, 2454-2462 (2014). 
10. Li, J., Jiang, Z., Hu, L. \& Hu, C. Selective conversion of cellulose in corncob residue to levulinic acid in an aluminum trichloridesodium chloride system. Chem Sus Chem 7, 2482-2488 (2014).

11. Jae, J. et al. Depolymerization of lignocellulosic biomass to fuel precursors: maximizing carbon efficiency by combining hydrolysis with pyrolysis. Energy Environ. Sci. 3, 358-365 (2010).

12. Hakola, M. et al. Liberation of cellulose from the lignin cage: A catalytic pretreatment method for the production of cellulosic ethanol. Chem Sus Chem 3, 1142-1145 (2010).

13. Grande, P. M. et al. Fractionation of lignocellulosic biomass using the OrganoCat process. Green Chem. 17, 3533-3539 (2015).

14. Delmas, M. Vegetal refining and agrochemistry. Chem. Eng. Technol. 31, 792-797 (2008).

15. vom Stein, T. et al. From biomass to feedstock: one-step fractionation of lignocellulose components by the selective organic acidcatalyzed depolymerization of hemicellulose in a biphasic system. Green Chem. 13, 1772-1777 (2011).

16. Bozell, J. J. \& Petersen, G. R. Technology development for the production of biobased products from biorefinery carbohydrates-the US Department of Energy's “Top 10" revisited. Green Chem. 12, 539-554 (2010).

17. Datta, R. \& Henry, M. Lactic acid: recent advances in products, processes and technologies-a review. J. Chem. Technol. Biot. 81, $1119-1129$ (2006).

18. Singhvi, M. \& Gokhale, D. Biomass to biodegradable polymer (PLA). RSC Adv. 3, 13558-13568 (2013).

19. Gao, C., Ma, C. \& Xu, P. Biotechnological routes based on lactic acid production from biomass. Biotechnol. Adv. 29, 930-939 (2011).

20. Maki-Arvela, P., Simakova, I. L., Salmi, T. \& Murzin, D. Y. Production of lactic acid/lactates from biomass and their catalytic transformations to commodities. Chem. Rev. 114, 1909-1971 (2014).

21. Maas, R. H. et al. Lactic acid production from lime-treated wheat straw by Bacillus coagulans: neutralization of acid by fed-batch addition of alkaline substrate. App. Microbiol. Biotechnol. 78, 751-758 (2008).

22. Kong, L., Li, G., Wang, H., He, W. \& Ling, F. Hydrothermal catalytic conversion of biomass for lactic acid production. J. Chem. Technol. Biot. 83, 383-388 (2008).

23. Dusselier, M., Van Wouwe, P., Dewaele, A., Makshina, E. \& Sels, B. F. Lactic acid as a platform chemical in the biobased economy: the role of chemocatalysis. Energy Environ. Sci. 6, 1415-1442 (2013).

24. Jin, F. \& Enomoto, H. Rapid and highly selective conversion of biomass into value-added products in hydrothermal conditions: chemistry of acid/base-catalysed and oxidation reactions. Energy Environ. Sci. 4, 382-397 (2011).

25. Younas, R. et al. Lactic acid production from rice straw in alkaline hydrothermal conditions in presence of NiO nanoplates. Catal. Today 274, 40-48 (2016).

26. Zhang, S., Jin, F., Hu, J. \& Huo, Z. Improvement of lactic acid production from cellulose with the addition of $\mathrm{Zn} / \mathrm{Ni} / \mathrm{C}$ under alkaline hydrothermal conditions. Bioresource Technol. 102, 1998-2003 (2011).

27. Wang, F., Huo, Z., Wang, Y. \& Jin, F. Hydrothermal conversion of cellulose into lactic acid with nickel catalyst. Rese. Chem. Intermediat. 37, 487-492 (2011).

28. Wang, Y. et al. Chemical synthesis of lactic acid from cellulose catalysed by lead(II) ions in water. Nat. Commun. 4, 2141-2148 (2013).

29. Lei, X., Wang, F., Liu, C., Yang, R. \& Dong, W. One-pot catalytic conversion of carbohydrate biomass to lactic acid using an $\mathrm{ErCl}_{3}$ catalyst. Appl. Catal. A: Gen. 482, 78-83 (2014).

30. Yang, L. et al. Catalytic conversion of hemicellulosic biomass to lactic acid in $\mathrm{pH}$ neutral aqueous phase media. Appl. Catal. B: Environ. 162, 149-157 (2015).

31. Maki-Arvela, P., Salmi, T., Holmbom, B., Willfor, S. \& Murzin, D. Y. Synthesis of sugars by hydrolysis of hemicelluloses-a review. Chem. Rev. 111, 5638-5666 (2011).

32. Xing, R., Qi, W. \& Huber, G. W. Production of furfural and carboxylic acids from waste aqueous hemicellulose solutions from the pulp and paper and cellulosic ethanol industries. Energy Environ. Sci. 4, 2193-2205 (2011).

33. Luo, Y., Hu, L., Tong, D. \& Hu, C. Selective dissociation and conversion of hemicellulose in Phyllostachys heterocycla cv. var. pubescens to value-added monomers via solvent-thermal methods promoted by $\mathrm{AlCl}_{3}$. RSC Adv. 4, 24194 (2014).

34. Kim, E. S., Liu, S., Abu-Omar, M. M. \& Mosier, N. S. Selective conversion of biomass hemicellulose to furfural using maleic acid with microwave heating. Energy Fuel. 26, 1298-1304 (2012).

35. Xing, R. et al. Production of jet and diesel fuel range alkanes from waste hemicellulose-derived aqueous solutions. Green Chem. 12, 1933-1946 (2010).

36. Hu, L. et al. The degradation of the lignin in Phyllostachys heterocycla cv. pubescens in an ethanol solvothermal system. Green Chem. 16, 3107-3116 (2014).

37. Wen, J., Sun, S., Xue, B. \& Sun, R. Recent advances in characterization of lignin polymer by solution-state nuclear magnetic resonance (NMR) methodology. Materials 6, 359-391 (2013).

38. Lee, J. W., Rodrigues, R. C., Kim, H. J., Choi, I. G. \& Jeffries, T. W. The roles of xylan and lignin in oxalic acid pretreated corncob during separate enzymatic hydrolysis and ethanol fermentation. Bioresource Technol. 101, 4379-4385 (2010).

39. Scordia, D., Cosentino, S. L. \& Jeffries, T. W. Effectiveness of dilute oxalic acid pretreatment of Miscanthus x giganteus biomass for ethanol production. Biomass Bioenerg. 59, 540-548 (2013).

40. Oh, S. Y. et al. Crystalline structure analysis of cellulose treated with sodium hydroxide and carbon dioxide by means of X-ray diffraction and FTIR spectroscopy. Carbohyd. Res. 340, 2376-2391 (2005).

41. Kootstra, A. M. J., Beeftink, H. H., Scott, E. L. \& Sanders, J. P. M. Optimization of the dilute maleic acid pretreatment of wheat straw. Biotechnol. Biofuel. 2 (2009).

42. Tan, S. S. Y. et al. Extraction of lignin from lignocellulose at atmospheric pressure using alkylbenzenesulfonate ionic liquid. Green Chem. 11, 339-345 (2009).

43. Jiang, Z., Yi, J., Li, J., He, T. \& Hu, C. Promoting effect of sodium chloride on the solubilization and depolymerization of cellulose from raw biomass materials in water. Chem Sus Chem 8, 1901-1907 (2015).

44. Chandel, A. K. et al. Ultra-strucural mapping of sugarcane bagasse after oxalic acid fiber expansion (OAFEX) and ethanol production by Candida shehatae and saccharomyces cerevisiae. Biotechnol. Biofuel. 6 (2013).

45. Kamiyama, Y. \& Sakai, Y. Rate of hydrolysis of xylo-oligosaccharides in diltue $\mathrm{H}_{2} \mathrm{SO}_{4}$. Carbohyd. Res. 73, 151-158 (1979).

46. Liu, Z. et al. Conversion of biomass-derived carbohydrates to methyl lactate using solid base catalysts. Catal. Commun. 15, 82-87 (2011).

47. Aida, T. M., Shiraishi, N., Kubo, M., Watanabe, M. \& Smith, R. L. Reaction kinetics of d-xylose in sub- and supercritical water. J. Supercrit. Fluid. 55, 208-216 (2010).

48. Wang, A. Q. \& Zhang, T. One-pot conversion of cellulose to ethylene glycol with multifunctional tungsten-based catalysts. Accounts Chem. Res. 46, 1377-1386 (2013).

49. Qi, W. Y. et al. Catalytic pyrolysis of several kinds of bamboos over zeolite NaY. Green Chem. 8, 183-190 (2006).

\section{Acknowledgements}

This work is financially supported by the National Basic Research Program of China (973 program, No. 2013CB228103). The characterization of the residue from Analytical and Testing Center of Sichuan University are greatly appreciated. 


\section{Author Contributions}

T. He, J.M. Li and C.W. Hu conceived and designed the experiments. T. He and Z.C. Jiang performed the experiments. All co-authors participated in the process of data analysis and the writing of the paper.

Additional Information

Supplementary information accompanies this paper at http://www.nature.com/srep

Competing financial interests: The authors declare no competing financial interests.

How to cite this article: He, T. et al. Fractionation for further conversion: from raw corn stover to lactic acid. Sci. Rep. 6, 38623; doi: 10.1038/srep38623 (2016).

Publisher's note: Springer Nature remains neutral with regard to jurisdictional claims in published maps and institutional affiliations.

(c) (i) This work is licensed under a Creative Commons Attribution 4.0 International License. The images or other third party material in this article are included in the article's Creative Commons license, unless indicated otherwise in the credit line; if the material is not included under the Creative Commons license, users will need to obtain permission from the license holder to reproduce the material. To view a copy of this license, visit http://creativecommons.org/licenses/by/4.0/

(c) The Author(s) 2016 